\title{
Editorial
}

\section{Radiation Effects in Solar Cells and Optoelectronic Devices}

\author{
Aleksandra Vasic, ${ }^{1}$ Predrag Osmokrovic, ${ }^{2}$ Nenad Marjanovic, ${ }^{3}$ and Momčilo Pejovic ${ }^{4}$ \\ ${ }^{1}$ Faculty of Mechanical Engineering, University of Belgrade, Kraljice Marije 16, 11000 Belgrade, Serbia \\ ${ }^{2}$ Faculty of Electrical Engineering, University of Belgrade, Bulevar kralja Aleksandra 73, 11000 Belgrade, Serbia \\ ${ }^{3}$ CSEM Centre Suisse d'Electronique et de Microtechnique SAEntwicklungszentrum für Polytronics, Tramstrasse 99, \\ 4132 Muttenz, Switzerland \\ ${ }^{4}$ Faculty of Electronic Engineering, University of Niš, Aleksandra Medvedeva 14, $18000 \mathrm{Nišs}$, Serbia \\ Correspondence should be addressed to Aleksandra Vasic; avasic@mas.bg.ac.rs
}

Received 25 August 2013; Accepted 25 August 2013

Copyright (C) 2013 Aleksandra Vasic et al. This is an open access article distributed under the Creative Commons Attribution License, which permits unrestricted use, distribution, and reproduction in any medium, provided the original work is properly cited.

Beside the diversity of the device technologies used for designing the solar cells and various optoelectronic devices, there are a variety of radiation environments in which they are used (natural space and atmospheric, as well as military and civil nuclear environments, etc.). Reliability of electrical devices in a radiation environment is very important, and extensive studies concerning the development of semiconductor devices that can operate normally in such conditions have been seriously undertaken. Possible degradation of the electrical performance of optoelectronic devices in general, induced by irradiation, means that very strict conditions for their application must be predetermined for the worst case scenario. Performance failure in such conditions could have negative impact on both the financial and environmental aspects of the device application.

The lifetime of the semiconducting device is restricted by the degree of radiation damage that the device receives. This is an important factor that affects the performance of various devices in practical applications. The permanent damage in the materials is caused by collisions of the incident radiation particles with the atoms in the crystalline lattice, which are displaced from their positions. These defects degrade the transport properties of the material and particularly the minority carrier lifetime. This lifetime decrease produces degradation of the parameters of the device ultimately leading to an increase of the noise level. The interaction between vacancies, self-interstitials, impurities, and dopants in the basic material leads to the formation of undesirable point defects such as recombination and compensator centers which affect performance of the solar cells and other optoelectronic devices, especially in space. Introduction of radiation-induced recombination centers reduces the minority carrier lifetime in the base layer of the $p$-n junction increasing series resistance. After very high doses of radiation series resistance of the base layer could be so high that most of the power generated by the device such as solar cell is dissipated by its own internal resistance.

On the other hand, $\gamma$ radiation damage is induced by the ionization and excitation of the atoms within the junction space charge region of a junction device such as solar cell. The presence of impurity atoms that are either added to the base material as donors, or during the manufacturing process, has indicated the possibility that some of the produced electrons might be trapped by those atoms between the valence and conduction band. As a consequence, the output of the optoelectronic device exposed to $\gamma$ radiation is also reduced. Therefore, from the technological point of view, it is important to study the variations induced by irradiation of semiconductor junction characteristic parameters (ideality factor, saturation current, etc.) that affect the performance of the solar cells, photodiodes, phototransistors, and other photonic devices.

This special issue reveals recent developments in the vastly undertaken investigations concerning radiation effects in various optoelectronic devices (solar cells, photodiodes, phototransistors, insulating layers, memories, dosimeters, etc.). Each paper was reviewed by at least two reviewers 
(mostly three) and presents the most recent breakthroughs in this interesting field.

Many fundamental and practical problems were discussed in the received and accepted papers, ranging from solar cells, photodiodes, phototransistors, insulating films, memories, and so forth. Various experimental techniques, numerical analysis, and theoretical discussions were presented in order to reveal current problems, and to offer adequate solutions. Behavior of the solar cells, photodiodes, and photodetectors exposed to gamma and neutron irradiation was discussed in D. Nikolic et al.'s and B. Simic et al.'s papers, respectively. After thorough investigations, the authors concluded that although neutron irradiation in all samples caused degradation of their structure and deterioration of the output characteristics, solar panels have proved to be most resistant to the effects of neutrons. Also, results obtained from gamma irradiation showed the degradation of the $I$ $V$ characteristics, but following annealing process improved these characteristics. Due to their amplifying action, the phototransistors are the most sensitive to radiation effects. On the other hand, the solar panels are the least sensitive to gamma radiation.

Another vast group of electrical devices such as resistors were considered in the I. Kostic et al.s paper. The temperature dependence has been treated without taking the thermal source into consideration, which means that, in the cosmic radiation conditions, this dependence could be reached by the collision energy transfer between particle from cosmic radiation and the observed component. A novel approach was proposed for thermal testing of the resistors. It was found that thermography can be used as noncontact method because it gives temperature distribution in more than a couple points and because in the short time a set of measurements can be done.

Radiation effects in insulating films were also investigated. In L. Timotijevic et al.s paper Monte Carlo simulation of proton beam effects on five insulating materials, commonly encountered in modern day electronic components: silicon dioxide, silicon nitride, aluminum nitride, alumina, and polycarbonate were discussed. It was found that nonionizing energy loss of high-energy protons is low, and they traverse the films without much atomic displacement. However, for the lower part of the investigated proton energy range (from $10 \mathrm{keV}$ to $1 \mathrm{MeV}$ ), substantial ionization losses and NIEL are to be expected. Ionization and displacement damage produced by protons could influence the properties of these insulators and compromise their reliability within complex structures and devices.

A newly discovered class of materials, Cooper-pair insulators, based on $\mathrm{InO}_{x}$ films, with distinct structural properties and unique physical mechanism of electrical current conduction were investigated in S. Milosavljevic et al.s paper. The study suggests that radiation-induced changes in $\mathrm{InO}_{x}$ films exposed to ion beams could significantly alter their currentvoltage characteristics, and that a transition to a metallic state is possible, due to radiation-induced perturbation of the fine-tuned granular structure. Furthermore, incident and displaced ions can break up enough Cooper pairs in $\mathrm{InO}_{x}$ films to cause dissolution of this specific insulating state.
Since Cooper-pair insulators are recently used in resistive random-access memories, investigation of effects of radiation environment on different memory devices is of considerable relevance. In I. Fetahovic et al.s paper, different types of commercially available memory chips were exposed to indirect ionizing radiation by changing radiation dose intensity. The effect of direct ionizing radiation on semiconductor memory behavior has been analyzed by using Monte Carlo simulation method. Obtained results showed that gamma radiation causes decrease in threshold voltage, being proportional to the absorbed dose of radiation. Monte Carlo simulations of radiation interaction with material proved to be significant and can be a good estimation tool in probing semiconductor memory behavior in radiation environment.

Another type of commonly used memories, such as flash memories exposed to radiation, was investigated in B. Cavric et al's paper. This topic is up-to-date since the high degree of components miniaturization integrated into the flash memory causes the extreme sensitivity of this memory type on the ionizing radiation effects. The effects of ionizing radiation may cause changes in stored data, or even the physical destruction of the components. The obtained results indicate that the sensitivity to gamma rays depends primarily on the thickness of the dielectric layers of cells, which is about the same for all tested memories, since they belong to the same generation of technology. Partial recovery of errors caused by radiation, in a few weeks at room temperature, was observed in all the four types of memory.

One group of semiconducting devices that are by their function always exposed to radiation, dosimeters, were the topic of M. Pejovic et al's paper. Specifically, radiationsensitive p-channel MOSFETs (also known as RADFETs) have recently been developed for applications in space, nuclear industry research, and radiotherapy, so sensitivity of $\mathrm{p}$-channel MOSFET to $\mathrm{X}$ and gamma irradiation is of great interest. Results have shown that the response is greater when RADFETs are irradiated with X-rays. It was concluded that this is a consequence of the dominant influence of photoelectric effect in X-ray cases in comparison to Compton's effect in gamma-ray cases. Another significant conclusion was that a satisfactory response of these RADFETs could be achieved for lower doses especially in X-ray cases, which would enable their efficient application for measuring low doses order of several $\mathrm{mGy}$ used in diagnostic and interventional radiology.

All of the presented papers give a substantial insight into the state of the art concerning this important interdisciplinary field. Since very comprehensive comparative analysis of measurement results has been performed in order to determine the reliability of optoelectronic devices in radiation environment, the editorial team expects that these papers would inspire further investigations and possible breakthroughs in this important area.

\section{Acknowledgments}

We would like to thank all contributing authors, reviewers, and Editorial Board of the International Journal of Photoenergy for their support and efforts to make this special 
issue possible. Also, the support of Safi Hosni, Editorial Office, Hindawi Publishing Corporation, was invaluable for preparing this issue, for which we are thankful.

Aleksandra Vasic Predrag Osmokrovic Nenad Marjanovic Momčilo Pejović 

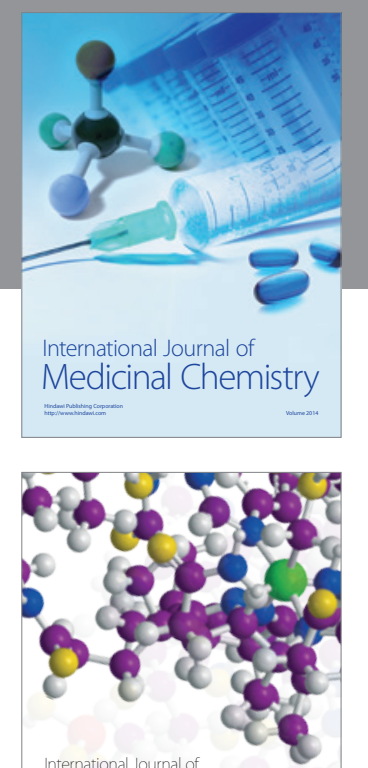

\section{Carbohydrate} Chemistry

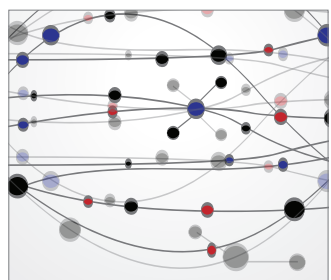

The Scientific World Journal
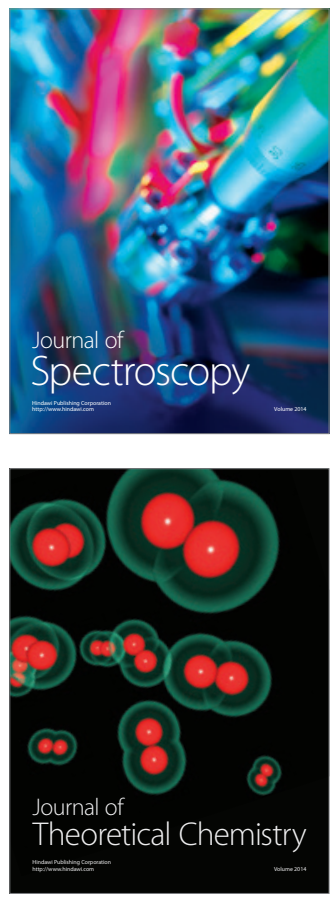
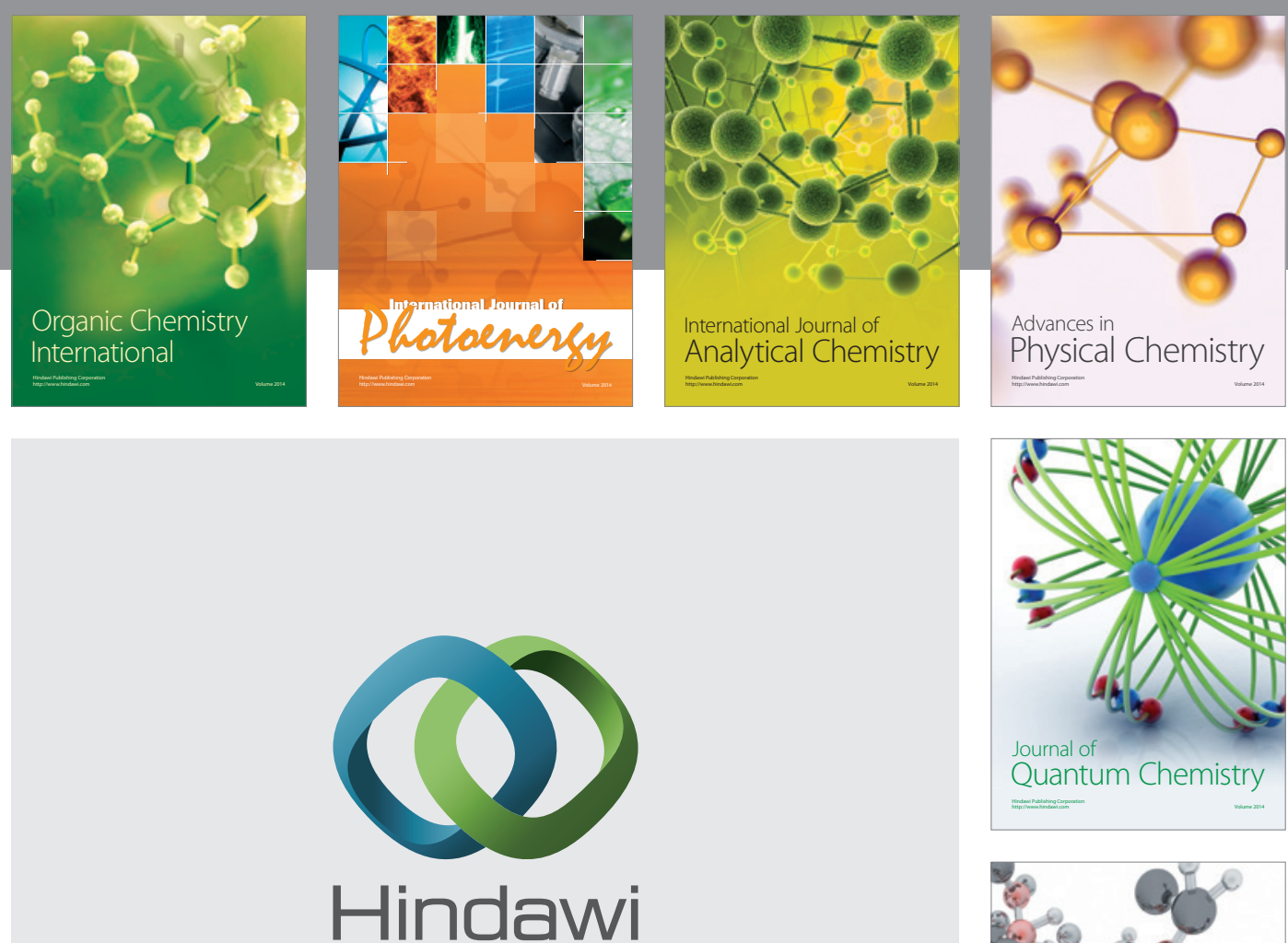

Submit your manuscripts at

http://www.hindawi.com

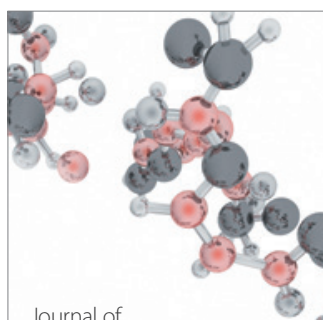

Analytical Methods

in Chemistry

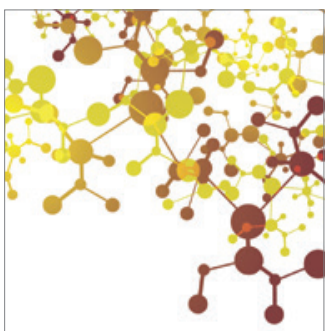

Journal of

Applied Chemistry

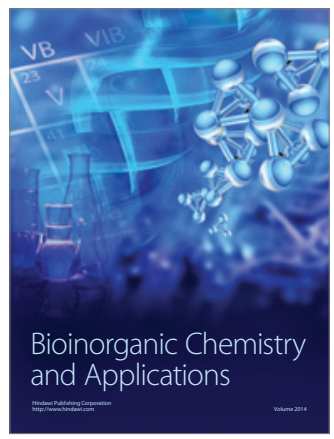

Inorganic Chemistry
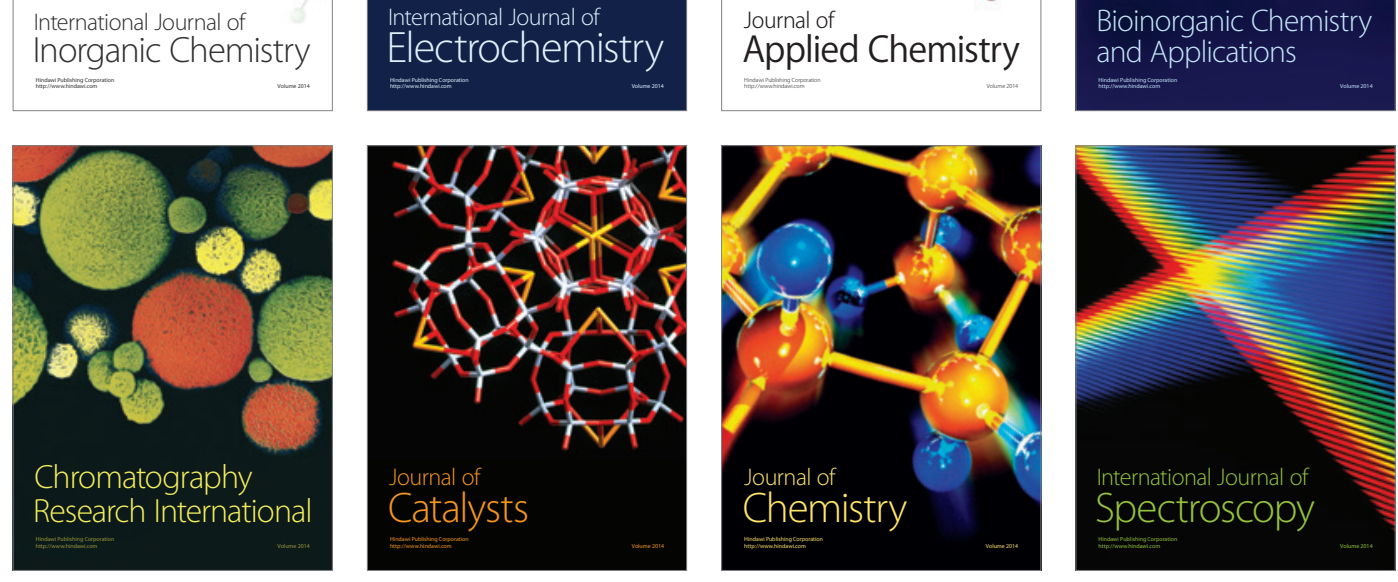